\title{
SLEEP AND SEX \\ THE RELATION BETWEEN SLEEP DISTURBANCES AND SEXUAL FUNCTION
}

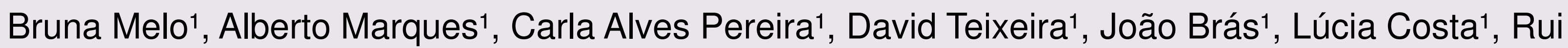
Sousa ${ }^{1}$, Elsa Monteiro ${ }^{1}$

1Department of Psychiatry and Mental Health, Centro Hospitalar Tondela-Viseu, EPE., Viseu, Portugal

\section{Objectives}

To present a non-systematic review of the literature on the relationship between sleep disorders and sexual dysfunction.

\section{Background and aims}

Sleep and Sex are ubiquitous phenomenon in animal behavior, both deeply tied to endocrine function.

The relationship between mental health and sleep, and mental health and sexual function are well established. Sexual dysfunction is a frequent multifactorial problem, and psychiatric disorders are one of its main causes. In turn, sleep disorders also have a very high prevalence and varied causes. Despite this, the direct relation of sleep disorders to sexual disorders is relatively unexplored.

\section{Materials and methods}

A literature search was conducted using the search engine Pubmed $\AA$ and the keywords sleep disorders and sexual dysfunction.

\section{Conclusions}

There seems to be a link between sleep and sexual dysfunction that is beyond the shared influence of other mental health factors. Given the intricate relationship between these two vital dimensions, dysfunctions in one area should lead to the investigation of dysfunctions in the other.

References:

1. Amasyali AS, Ta E, Amasyali SY, Turan Y, Kazan E, Sari E, et al. Effects of low sleep quality on sexual function, in women with fi bromyalgia. 2015;(February):1-4.

2. Mong JA, Cusmano DM, Mong JA. Sex differences in sleep : impact of biological sex and sex steroids. 2016;

3. Seehuus M, Pigeon W. The sleep and sex survey: Relationships between sexual function and sleep. J Psychosom Res [Internet]

2018:112(June):59-65. Available from: https://doi.org/10.1016/.jpsychores.2018.07.005

4. Cho JW, Duffy JF. Sleep, Sleep Disorders , and Sexual Dysfunction. 2018;1-15.
The relationship between sleep apnea and erectile dysfunction (ED) is well known; however, other sleep disturbances are infrequently referred to as cause or consequence of sexual dysfunction.

Some studies have shown that insomnia is related to ED and has been found to be an independent risk factor related to sexual dysfunction (SD).

It was found that sleep disorder had a negative effect on sexual function and may be the leading cause of SD among women with Fibromyalgia. not yet fully understood, it seems to be related both to psychogenic issues and to a neuroendocrine dysregulation, with decreased testosterone and progesterone levels, due to the reduction of sleep duration.

\section{"Insomnia has been found to be an independent risk factor of sexual dysfunction."}

Moreover, since both problems most often have the same temporal and spatial location (at night and in bed), there is probably a behavioral association between the two.
Although the mechanism involved in this relationship is 